\title{
The Bond Graph Method for Analysis of the Micro-Motion Characteristics of a Micro Gripper
}

\author{
Chao Lin ${ }^{1, *}$ - Yi-hang Ren ${ }^{1}$ - Jiu-xiang Ji1 - Li-zhong Cai ${ }^{1}$ - Ji-ming Shao ${ }^{2}$ \\ ${ }^{1}$ Chongqing University, The State Key Laboratory of Mechanical Transmission, China \\ ${ }^{2}$ Shanghai Key Laboratory of Spacecraft Mechanism, China
}

\begin{abstract}
A full-flexure micro gripper with three-stage amplification has been designed. The pseudo-rigid-body (PRB) model of a bridge-type amplification mechanism (BTAM) and micro gripper are established; the input/output stiffness of the BTAM are deduced on the use of the compliant mechanism, Castigliano's theorem and the PRB method. The characteristic equations and state-space equations of the micro gripper are derived on the basis of bond graph theory. The displacement simulation curve and flexible hinge angular simulation curve of the micro gripper are acquired through Matlab R2013b programming. Ansys13.0 finite element simulation software is utilized for simulation analysis of the micro gripper micro-motion. The micro gripper is $3 D$ printed using laser rapid prototyping technology, and a test bench has been set up. The experimental value, finite element analysis value, and Matlab simulation value are comparatively analysed, and the change rules are essentially the same. As a result, the validity of the bond graph model of the micro gripper is verified, and providing a new effective method for the flexible mechanism analysis.
\end{abstract}

Keywords: micro gripper, pseudo-rigid-body (PRB), flexure hinge, bond graph, Matlab R2013b, 3D print

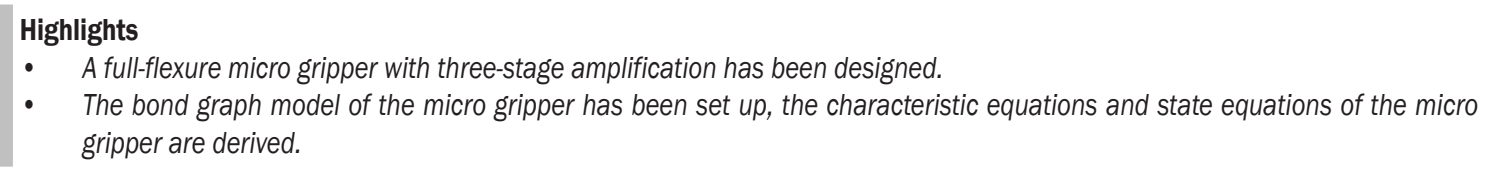

\section{INTRODUCTION}

In recent years, with the rapid development of science and technology, micro/nanotechnology has been becoming a key direction in research around the world. The demand for micro-motion devices has increased in many industrial applications, particularly in the fields of manipulating biological cells, microsurgical operation and assembling micro-machines. With the operation objects trending towards miniaturization, developing flexible, high precision and easily control microrobots are becoming an urgent requirement, and micro-manipulating robots combined with micropositioning technology and robot technology are receiving increased attention [1] and [2]. According to the driving principle, micro grippers can be roughly divided into five categories: electrothermal actuators [3], electrostatic actuators [4], piezoelectric actuators (PZTs) [5], shape memory alloy (SMA) actuators [6], and electromagnetic actuators [7]. PZTs are widely used because they have many advantages such as stable output displacement, great output force, high resolution, high response speed and are easy to control [8] and [9]. Nah and Zhong [9] designed a monolithic compliant-flexure-based micro gripper, of which the displacement amplification and maximum stroke are $3.0 \mu \mathrm{m}$ and $170 \mu \mathrm{m}$, respectively. Zubir et al. [10] developed a compliant-based micro gripper for high precision manipulation; a high displacement amplification and a maximum stroke of $100 \mu \mathrm{m}$ can be achieved. Ramadan et al. [11] proposed a chopstick-like two-fingered micromanipulator based on a hybrid mechanism for cell manipulation. Xiao et al. [12] presented a micro gripper which has absolutely parallel movement of the gripping arms; the displacement amplification ratio is about $10, \mu \mathrm{m}$, and the micro gripper arm maximum stroke is $300 \mu \mathrm{m}$. Yu et al. [13] studied a piezoelectric-based micro gripper of which the displacement amplification of one side can be 10.6, and the maximum output displacement is $261 \mu \mathrm{m}$. Furthermore, other micro grippers have been designed, such as a thin-walled copper tube by Li et al. [14], a compliant piezoelectric actuator based micro gripper by Jain et al. [15], a single active finger ionic polymer metal composite (IPMC) micro gripper by Ford et al. [16], a rotary micro gripper with locking function by Hao et al. [17], a piezoelectric actuator for micro gripper by Jain et al. [18], and a piezoelectricactuated micro gripper with a three-stage flexurebased amplification by Wang et. al [19].

The transmission of flexible micro gripper motion depends on the deformation of the material, so the kinematic modelling accuracy is difficult to guarantee. Furthermore, it is difficult to obtain the specific state change of a single internal component of the micro gripper. The bond graph is put forward by Paynter, describing the transmission, storage, conversion and consumption of power [20]. The nonlinearity of 
a)

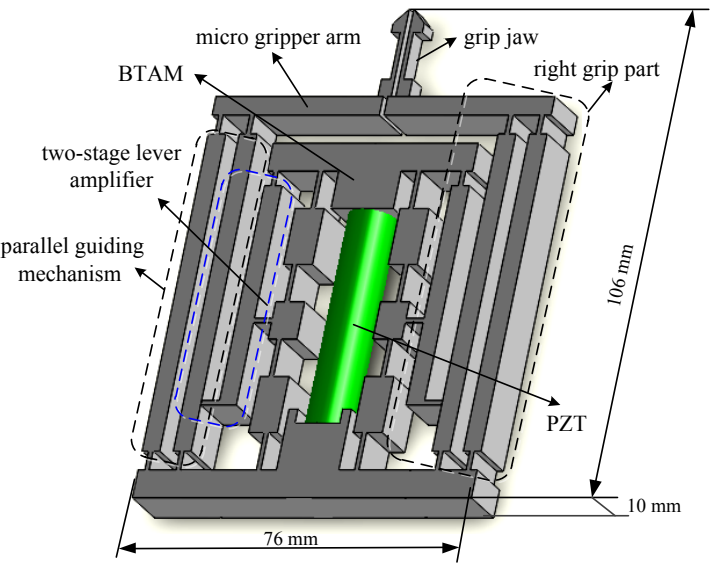

b)

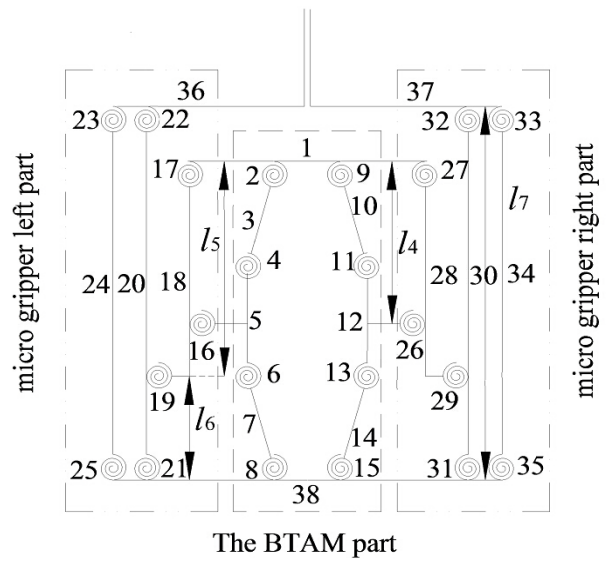

Fig. 1. Schematic diagram of micro gripper; a) geometrical model, and b) PRB model

systems can be intuitively considered in the bond graph method, and state variables in the model are all physical variables; thus, the state changes of the system can be further described. Moreover, the superiority of consistency between the bond graph and systematic state equations is significant. The corresponding mathematical model can be acquired by the bond graph of the system [21]. At present, bond graph modelling has been applied in the analysis of the Stewart platform mechanism [22]; however, the use of bond graph theory to analyse the flexible mechanism remains to be further developed.

Given the advantages of the bond graph in complex system physical model analysis, the bond graph model of the micro gripper is built after the analysis of working principles of the flexible micro gripper, stiffness of the bridge type amplification mechanism (BTAM) and parallel-guiding mechanism (PGM). The characteristic equations and statespace equations are derived. Finally, the output displacement simulation curve and flexible hinge angular displacement simulation curve of the micro gripper are obtained by using Matlab simulation and analysis.

\section{STRUCTURE DESIGN AND WORKING PRINCIPLE ANALYSIS OF THE MICRO GRIPPER}

According to the basic theory of compliant mechanism and mechanical principles, a micro gripper mechanism with large output displacement is designed (as shown in Fig. 1). The dimension is $106 \mathrm{~mm} \times 76 \mathrm{~mm} \times 10 \mathrm{~mm}$. The micro gripper is driven by PZTs; the input displacement provided by PZTs is amplified by BTAM, and further enlarged by a two-stage flexible lever. As a result, the micro gripper arm has a large stroke. Meanwhile, the parallelguiding mechanism ensures gripper arm maintains parallel movement and makes the grip jaw clip object more stably and firmly.

\section{STATICS ANALYSIS OF THE MICRO GRIPPER}

\subsection{Input Stiffness of the Micro Gripper}

According to the PRB method, a BTAM can be divided into 16 components, and each component is connected by a flexure hinge without clearance. The flexure hinge is equivalent to torsional spring, and the rest of the components are equivalent to a rigid rod. A quarter of the BTAM is taken for analysis because it is entirely symmetrical in structure. The PRB model and force analysis of BTAM are shown in Fig. 2.

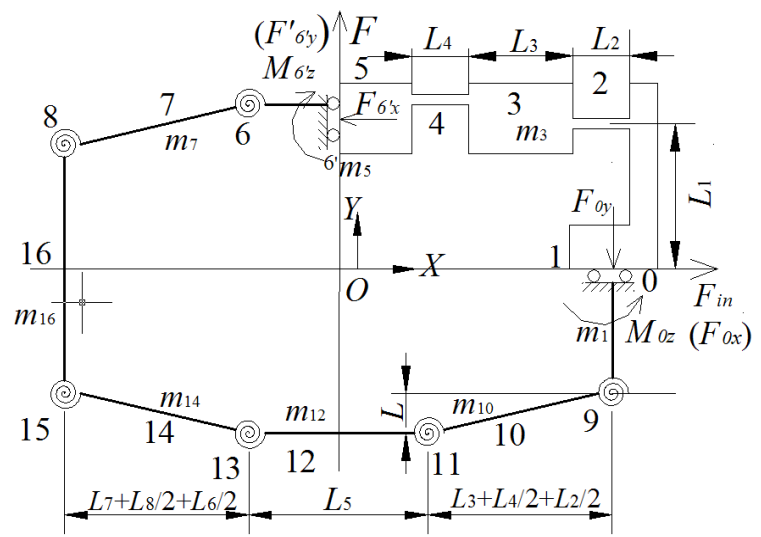

Fig. 2. Graph of PRB model and $1 / 4$ model force analysis of BTAM

Using Castigliano's theorem, input stiffness and output stiffness of BTAM were deduced by Lobontiu. The derivation process of input stiffness is the same as 
in reference [23], in this paper, however, in order to simplify the calculation, the shear force of flexure hinge is ignored. The output stiffness can be obtained in the same way while assuming the force $F_{6^{\prime} y}^{\prime}$ is applied to point $6^{\prime}$ when solving output stiffness. Flexible hinges 2 and 4 have the same dimension; hence, the compliance along the $\mathrm{x}$-axis and around the z-axis of flexible hinge 2 are equal to that of flexible hinge 2 correspondingly. That is, $C_{x-F}^{2}=C_{x-F}^{4}$, $C_{\theta-M}^{2}=C_{\theta-M}^{4}$.

Based on the analysis and theoretical derivation, the input stiffness and output stiffness of BTAM can be expressed by:

$$
\left\{\begin{array}{l}
k_{i n}=\frac{F_{0 x}}{u_{0 x}}=\frac{2}{4 C_{x-F}^{2}+L^{2} C_{\theta-M}^{2}} \\
k_{\text {out }}=\frac{F_{6^{\prime} y}^{\prime}}{u_{6^{\prime} y}}=\frac{2}{\left(L_{3}+L_{4}\right)^{2} C_{\theta-M}^{2}}
\end{array},\right.
$$

where $C_{\theta-M}^{2}=12 L_{2} /\left(E b t^{3}\right), C_{x-F}^{2}=L_{2} /(E t b), t, L$, and $L_{2}$ to $L_{5}$ are structural parameters of BTAM as shown in Fig. 2, $b$ is thickness of BTAM.

According to the movement of the micro gripper, when the micro gripper is driven by PZTs, not only the BTAM produces elongation deformation, flexible hinge 16, 19, 26 and 29 also achieve bending deformation in the driving force direction. As a consequence, the input stiffness of the micro gripper includes the input stiffness of BTAM and the bending stiffness of flexure hinge, as,

$$
K_{i n}=k_{i n}+\sum \frac{1}{C_{y-F}^{m}},
$$

where $m=16,19,26,29$, and $1 / C_{y-F}^{m}$ is bending stiffness of flexure hinge $\mathrm{m}$ when it is under the force $F_{6^{\prime}}$ which is parallel to the cross section, and $1 / C_{y-F}^{m}=E b t^{3} /\left(6 l^{2}\right), l$ is the length of flexure hinge.

\section{THE BOND GRAPH MODEL OF MICRO GRIPPER}

The energy conversion and transmission of interactional components are the basic foundations to set up the system bond graph in the engineering system. In the bond graph theory, the system with the co-existence of different forms of energy can be processed in a unified way; any system can be summarized into four state variables: flow variables $f(t)$, effort variables $e(t)$, generalized momentum $p(t)$, and generalized displacement $q(t)$.

Based on the theory and method of the bond graph, combined with the characteristics of the micro gripper driver and motion transmission, firstly the bond graph models, respectively, of BTAM, leverage, and PGM are set up; then the bond graph model of each part to set up the bond graph model of the microclamp is assembled. In the process of setting up the bond graph model of PGM, the rotary angles of the flexible hinges of PGM are assumed to be the same. For BTAM, it works as one end fixed while the other stretches, but in the bond graph model, both ends are assumed to stretch.

According to the transmission characteristics of the system and the causal relationships among different parts, considering generalized displacement $q(t)$ of capacitive component and generalized momentum $p(t)$ of inertial component as the state variables, $e(t)$ and $f(t)$ as the effort and flow of each bond., numbering each key from 1 to 82, the bond graph model of the micro gripper was established. The model is displayed in Fig. 4.

In Fig. 4, '0' connection and ' 1 ' connection stand for serial and parallel energy connections; the arrows indicate energy flow directions of the system. $I, C$ and $R$ represent the inertial component, capacitive component, and resistive component, respectively. Transformer (TF) helps to convert one type of motion to another. $S e$ is driving force, $K_{i n}$ is input stiffness of the micro gripper, $k_{i}, M_{i}, J_{i}$ and $u_{i}$ represent stiffness, mass, the moment of inertia and damping coefficient, and the subscript label is the series number of parts in Fig. 1 b. $k^{\prime}=k_{m}=1 / C_{\theta-M}^{2} \quad(m=2,4,6,8,9,11,13,15)$ is bending stiffness of flexure hinge of BTAM, $k^{\prime \prime}=k_{n}=1 / C_{\theta-M}^{16} \quad(n=16,17,19,21,22,23,25,26,27$, $29,31,32,33,35)$ is bending stiffness of flexure hinge of PGM. $J^{\prime}=J_{j}(j=3,7,10,14)$ is the moment of inertia of the rigid rod of BTAM, $J^{\prime \prime}=J_{k}(k=18,20)$ is moment of inertia of the rigid rod of lever, $J^{\prime \prime \prime}=J_{p}$ ( $p=24,28,30,34)$ is the moment of inertia of the rigid rod of PGM. By mechanics of materials, $J=m l^{2} / 12$.

\section{THE ESTABLISHMENT OF THE STATE-SPACE EQUATIONS}

The motion state of the system can be described by a set of state variables. State-space equations are the time-varying physical quantities of the system's internal state. As seen in Fig. 4, there are 30 energystorage components in the system, including 16 capacitive components and 14 inertial components. In these components, $I_{63}$ and $I_{82}$ have differential causality, and the rest have integral causality. The BTAM has equivalent damping in the direction of a driving force, as do both the left and right parts of micro gripper; as a consequence, the system also has resistive components. From Fig. 4, characteristic equations can be obtained as follows. 
micro gripper left part

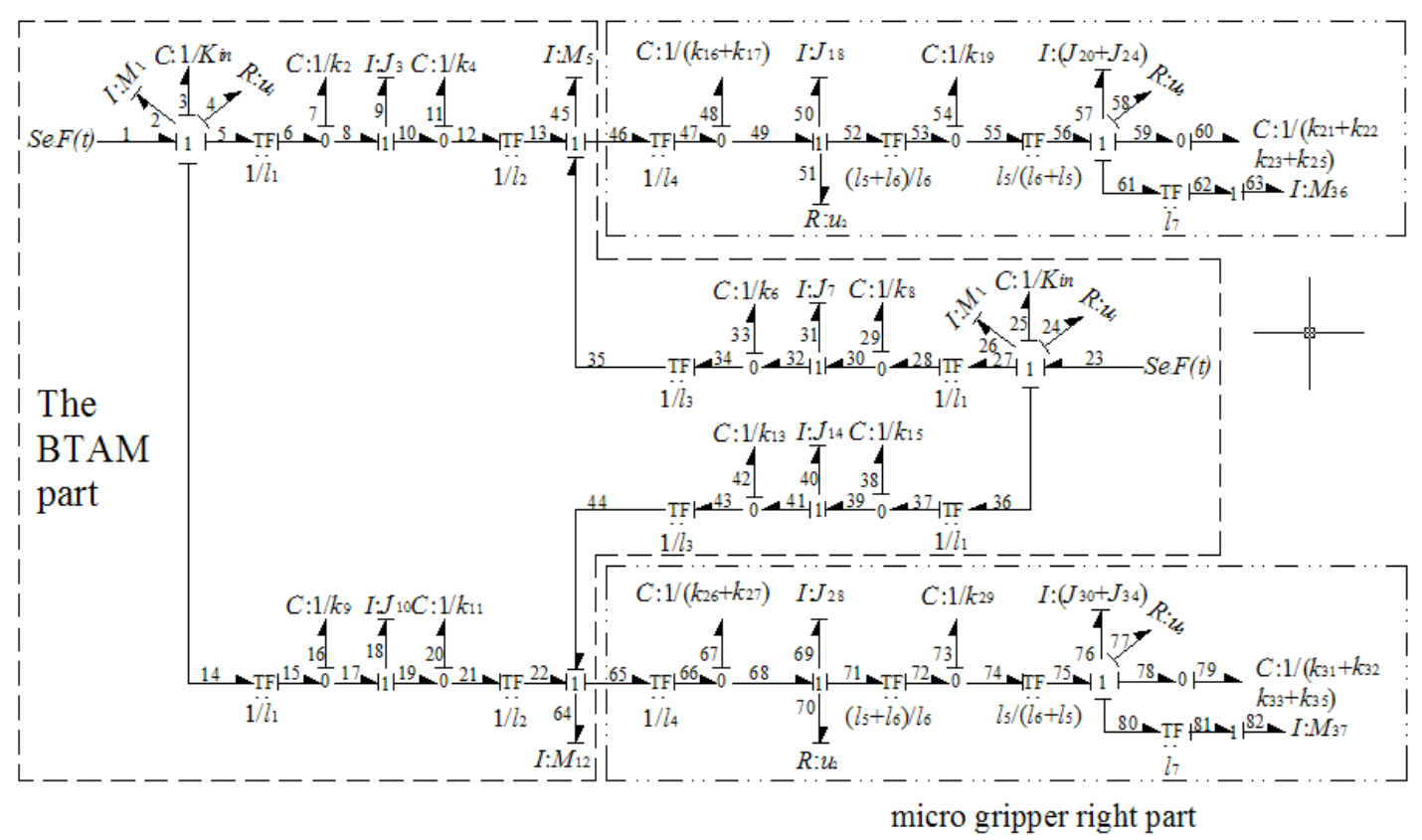

Fig. 3. The bond graph model of micro gripper

The characteristic equations of the resistive component are:

$$
\left\{\begin{array}{cl}
e_{4}=f_{4} R_{4}, & e_{58}=f_{58} R_{58} \\
e_{24}=f_{24} R_{24}, & e_{70}=f_{70} R_{70} . \\
e_{51}=f_{51} R_{51}, & e_{77}=f_{77} R_{77}
\end{array}\right.
$$

The characteristic equations of the energy-storage components with integral causality are:

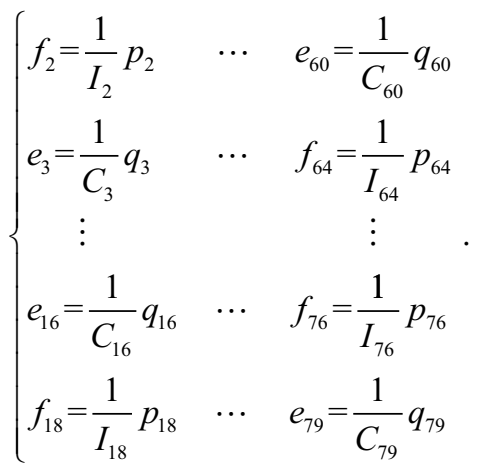

The characteristic equations of the energystorage components with differential causality are:

$$
\left\{\begin{array}{l}
e_{63}=I_{63} f_{63} \\
e_{82}=I_{82} f_{82}
\end{array} .\right.
$$

Assuming the system state variable is $\boldsymbol{X}=\left(\begin{array}{lllllll}p_{2} & q_{3} & q_{7} & \ldots & q_{70} & p_{73} & q_{75}\end{array}\right)_{1 \times 28}^{T}$, the subscript label of each state variable is the series number of bonds in Fig. 4. the state-space equations of a micro gripper can be deduced based on characteristic equations and characters of TF, connection ' 0 ' and ' 1 '. Since 28 state variables exist in Eq. (4), the statespace equations correspondingly contain 28 differential equations. The state-space equations can be derived as:

$$
\left\{\begin{array}{l}
\dot{p}_{2}=s e-e_{3}-e_{4}-e_{5}-e_{14}= \\
=s e-\frac{1}{C_{3}} q_{3}-R_{4} \frac{1}{I_{2}} p_{2}-\frac{1}{l_{1} C_{7}} q_{7}-\frac{1}{l_{1} C_{16}} q_{16} \\
\dot{q}_{3}=f_{2}=\frac{1}{I_{2}} p_{2} \\
\dot{q}_{7}=f_{6}-f_{8}=\frac{1}{l_{1} I_{2}} p_{2}-\frac{1}{I_{9}} p_{9} \\
\vdots \\
\dot{q}_{73}=f_{72}-f_{74}=\frac{l_{5}+l_{6}}{l_{6}} \frac{1}{I_{69}} p_{69}-\frac{l_{5}+l_{6}}{l_{5}} \frac{1}{I_{76}} p_{76} \\
\dot{p}_{76}=\frac{I_{76}}{I_{76}+l_{7}^{2} I_{82}}\left(e_{75}-e_{77}-e_{78}\right)= \\
=\frac{I_{73}}{I_{73}+l_{7}^{2} I_{78}}\left(\frac{l_{5}+l_{6}}{l_{5}} \frac{1}{C_{73}} q_{73}-R_{77} \frac{1}{I_{76}} p_{76}-\frac{1}{C_{79}} q_{79}\right) \\
\dot{q}_{79}=f_{78}=f_{76}=\frac{1}{I_{76}} p_{76}
\end{array}\right.
$$


In Eq. (3), the subscript labels of $I, C$ and $R$ are a series number of bonds, and $l_{1}=L, l_{2}=l_{3}=L_{3}+L_{4} / 2+$ $+L_{2} / 2, l_{5}$ and $l_{6}$ are structural parameters of leverPGM, $M$ is the quality of the translational components, and $M=m_{1}+m_{18}+m_{28}$, the subscript label is the series number of parts in Fig. 1b. $\mu$ is equivalent damping of BATM, $\mu_{1}$ is equivalent damping of lever- PGM mechanism.

\section{PROCESSING, SIMULATION, AND EXPERIMENT}

\subsection{Processing of the Micro Gripper}

Laser rapid prototyping technology an additive manufacturing processing method; also known as " $3 \mathrm{D}$ printing, it is a new manufacturing technology that integrates $\mathrm{CAD}, \mathrm{CNC}$, laser technology, automatic control technology, new materials and other advanced technologies. It has become widely used in machinery manufacturing, aerospace, medical and other fields. Because of the simple structure of the micro gripper, the $3 \mathrm{D}$ printing process of the micro gripper is more convenient. The micro gripper is processed through the EOS M280 laser molten sintering moulding machine, and separated from the base plate by using Wire EDM; the machining process is shown in Fig. 5. TC4 titanium alloy is chosen as the material of the micro gripper because of its good comprehensive properties, good plasticity and durability, and hightemperature deformation performance.
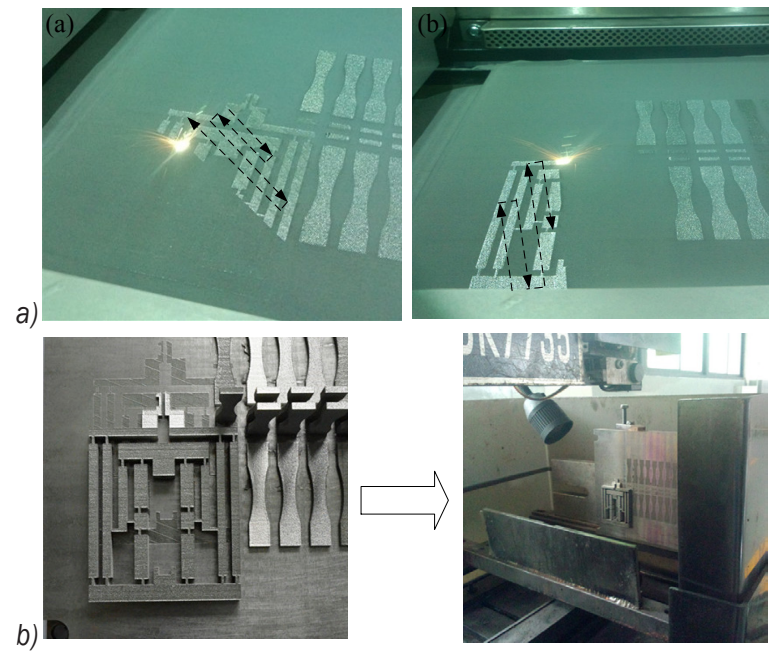

Fig. 4. Processing of the micro gripper; a) 3D printing of the micro gripper and b) wire EDM processing

\subsection{The Finite Element Simulation and Solution of State Equations}

The micro gripper is simulated and analysed with Ansys 13.0; the bottom is selected as fixed support, the mesh plots and working simulation diagram are shown in Fig. 5.

A variable order Adams PECE algorithm is a commonly used method for solving ordinary differential equations. It has the advantages of high precision, simple procedure, and stable calculation
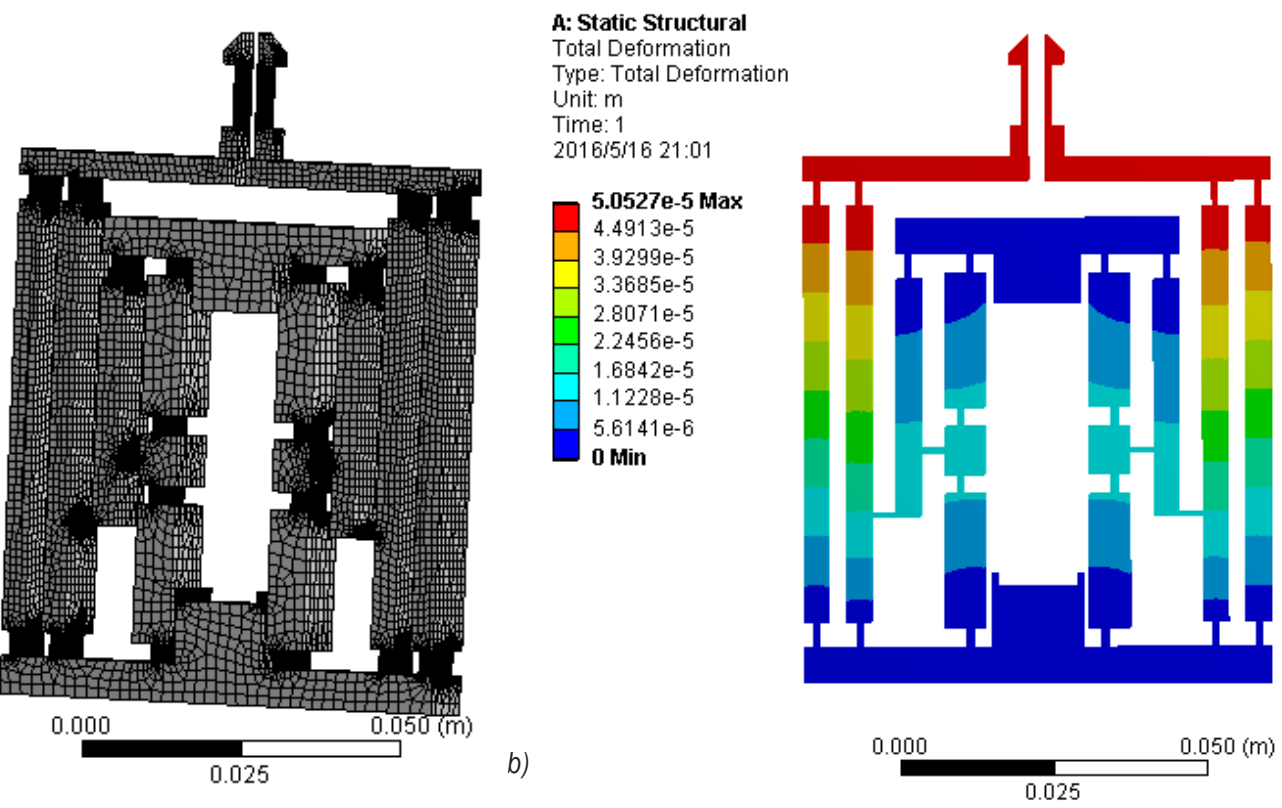

Fig. 5. The finite element simulation diagram of micro gripper; a) mesh plots, and b) motion simulation diagram 
Table 2. Key parameters of the micro gripper

\begin{tabular}{cccccccccc}
\hline$L[\mathrm{~mm}]$ & $L_{1}[\mathrm{~mm}]$ & $L_{2}[\mathrm{~mm}]$ & $L_{3}[\mathrm{~mm}]$ & $L_{4}[\mathrm{~mm}]$ & $L_{5}[\mathrm{~mm}]$ & $t[\mathrm{~mm}]$ & $b[\mathrm{~mm}]$ & $l_{4}[\mathrm{~mm}]$ & $l_{5}[\mathrm{~mm}]$ \\
\hline 1.5 & 11 & 3 & 22 & 3 & 4 & 1 & 10 & 30 & 40.4 \\
\hline$l_{6}[\mathrm{~mm}]$ & $l_{7}[\mathrm{~mm}]$ & $K_{\text {in }}[\mathrm{N} / \mathrm{mm}]$ & $k^{\prime}[(\mathrm{N} \cdot \mathrm{mm}) / \mathrm{rad}]$ & $\left.k^{\prime \prime}[\mathrm{N} \cdot \mathrm{mm}) / \mathrm{rad}\right]$ & $J^{\prime}\left[\mathrm{kg} \cdot \mathrm{mm}^{2}\right]$ & $J^{\prime \prime}\left[\mathrm{kg} \cdot \mathrm{mm}^{2}\right]$ & $J^{\prime \prime \prime}\left[\mathrm{kg} \cdot \mathrm{mm}^{2}\right]$ \\
\hline 19.6 & 72 & 88168 & 57680 & 21836 & 0.573 & 1.632 & 9.072 \\
\hline
\end{tabular}

Table 3. Relations output displacement and driving force of PZT of micro gripper

\begin{tabular}{lccccccccc}
\hline$\Delta L_{1}[\mu \mathrm{m}]$ & 1 & 2 & 3 & 4 & 5 & 6 & 7 & 8 & 9 \\
\hline$F_{\text {eff }}[\mathrm{N}]$ & 49.6 & 99.2 & 148.8 & 198.3 & 247.9 & 297.5 & 347.1 & 396.7 & 446.3 \\
\hline$\Delta L_{1}[\mu \mathrm{m}]$ & 10 & 11 & 12 & 13 & 14 & 15 & 16 & 17 & 18 \\
\hline$F_{\text {eff }}[\mathrm{N}]$ & 495.9 & 545.5 & 595 & 644.6 & 694.2 & 743.9 & 793.5 & 843.1 & 892.7 \\
\hline
\end{tabular}

process; its step size is easy to adjust. In comparison with the Runge-Kutta method, the variable order Adams PECE algorithm is a better method to perform the simulation analysis of the micro gripper, of which the range of allowable error is comparatively strict. Differential Eq. (15) is solved with the aid of the Matlab mathematical tool box in this paper. Because in the state equation represented by Eq. (6), the values of the inertial element, capacitive element, and resistive components are related to micro gripper parameters, the parameters of the micro gripper will be introduced before solving the state equation. The material of the micro gripper is TC4, the basic parameters of which are shown in Table 1. The key parameters of the micro gripper are shown in Table 2.

Table 1. Relevant parameter of material

\begin{tabular}{cccc}
\hline $\begin{array}{c}E \\
\begin{array}{c}\text { (elasticity modulus) } \\
{[\mathrm{GPa}]}\end{array}\end{array}$ & $\begin{array}{c}\mu \\
\text { (Poisson's ratio) }\end{array}$ & $\begin{array}{c}\rho \\
\text { (density) } \\
{\left[\mathrm{kg} / \mathrm{cm}^{3}\right]}\end{array}$ & $\begin{array}{c}\sigma_{s} \\
\text { (yield strength) } \\
{[\mathrm{MPa}]}\end{array}$ \\
\hline 206 & 0.28 & 7850 & 1176 \\
\hline
\end{tabular}

The PZT used here is 40VS15, which is made in China; its biggest driving force is $900 \mathrm{~N}$, the maximum displacement is $36 \mu \mathrm{m}$, the closed-loop resolution is $1.2 \mathrm{~nm}$, and stiffness is $15 \mathrm{~N} / \mu \mathrm{m}$. According to the stiffness characteristics of PZT, the relations between output force and output displacement of PZT under variable external loads can be obtained, shown in Table 3.

$\Delta L_{1}$ is the output displacement of the PZT under variable load; $F_{\text {eff }}$ is the output force of the PZT after overcoming the external loads.

The simulation curves of displacement of component 1 and angle displacement of flexible plate spring 21 within the scope of the biggest driving force are firstly obtained, the displacement simulation curves of component 1 are shown in Fig. 6.

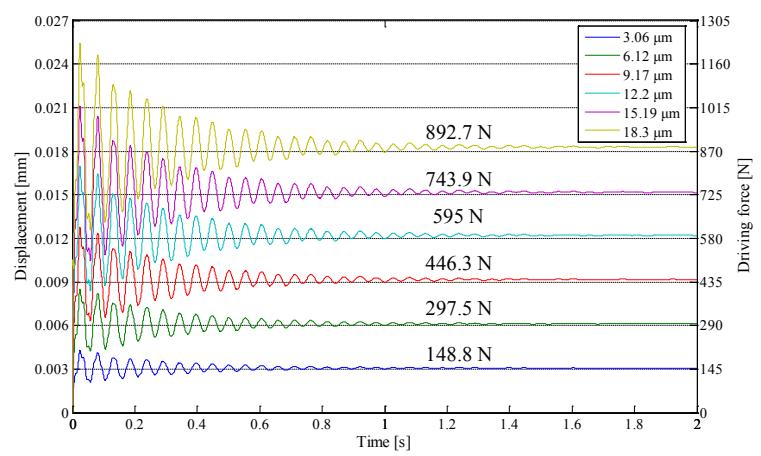

Fig. 6. Displacement simulation curves of component 1

The displacement simulation curves of component 1 under the force of $892.7 \mathrm{~N}, 743.9 \mathrm{~N}, 595$ $\mathrm{N}, 446.3 \mathrm{~N}, 297.5 \mathrm{~N}$ and $148.8 \mathrm{~N}$ are shown in Fig. 6 , and the final simulation displacement values are $18.3 \mu \mathrm{m}, 15.15 \mu \mathrm{m}, 12.2 \mu \mathrm{m}, 9.15 \mu \mathrm{m}, 6.11 \mu \mathrm{m}$ and $3.06 \mu \mathrm{m}$. Consequently, the fast response is crucial in microstructure [24] and [25]. It takes about $1.2 \mathrm{~s}$ for a steady response of component 1 .

In the same way, the angle displacement simulation curves of flexible plate spring 21 under the same input force can be obtained. Taking input force $148.8 \mathrm{~N}$ and $892.7 \mathrm{~N}$ as examples, the results are shown in Fig. 7.

The final angular simulation displacement values under $148.8 \mathrm{~N}$ and $892.7 \mathrm{~N}$ are $2.994 \times 10^{-4} \mathrm{rad}$, and $1.786 \times 10^{-3} \mathrm{rad}$ according to Fig. 7, The response time of the angular displacement of flexible plate spring 21 is about $3 \mathrm{~s}$. The length of the rod in parallel guides mechanism is $72 \mathrm{~mm}$, so the displacements of the gripping jaw can be approximately calculated to 21.53 

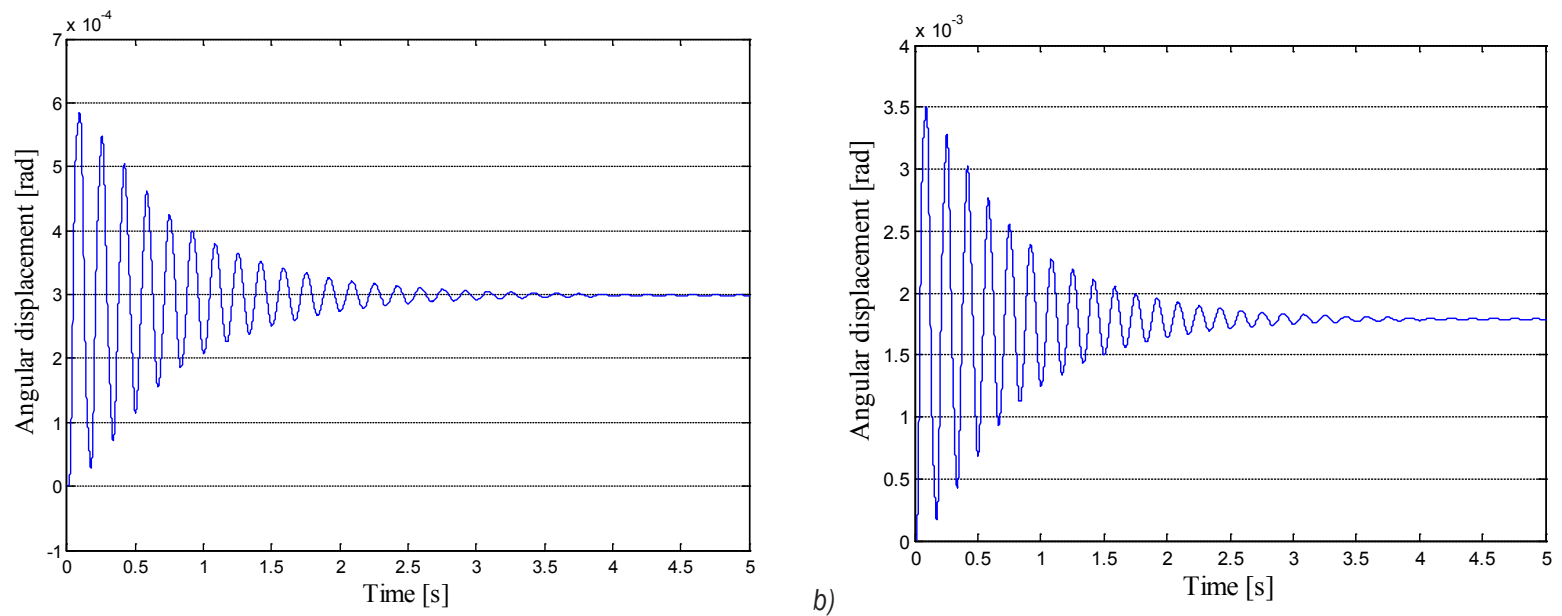

Fig. 7. Angular displacement simulation curves of flexible plate spring; under a) $148.8 \mathrm{~N}$, and b) $892.7 \mathrm{~N}$

$\mu \mathrm{m}$ and $128.81 \mu \mathrm{m}$. The angular displacement of the flexible spring plate 21 under input force ranges from $49.6 \mathrm{~N}$ to $829.7 \mathrm{~N}$ can be calculated with Simulink, and the output displacement of the micro gripper can be obtained.

\subsection{Experiments}

In order to verify the validity of the established bond graph model of the micro gripper, the displacement of the gripper arm (component 36) under different driving forces $\mathrm{F}$ needs to be tested and measured within a reasonable range. The experiment principle diagram is shown in Fig. 8.

The displacement measurement test bench of the micro gripper can be set up according to Fig. 8; it is shown in Fig. 9.

During the experiment, the PZT is driven by the PZT controller and the output displacement is 1 to 18 $\mu \mathrm{m}$, the displacement of gripper arm can be measured by the capacitance displacement sensor.

The finite element values, bond graph theoretic values and experimental values are compared; the results are obtained as shown in Fig. 10.

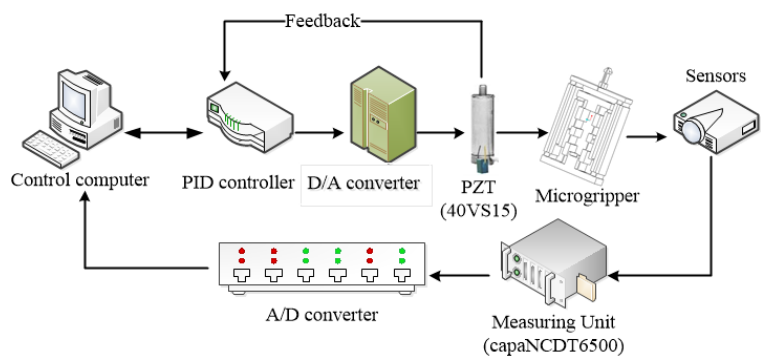

Fig. 8. Experiment principle diagram

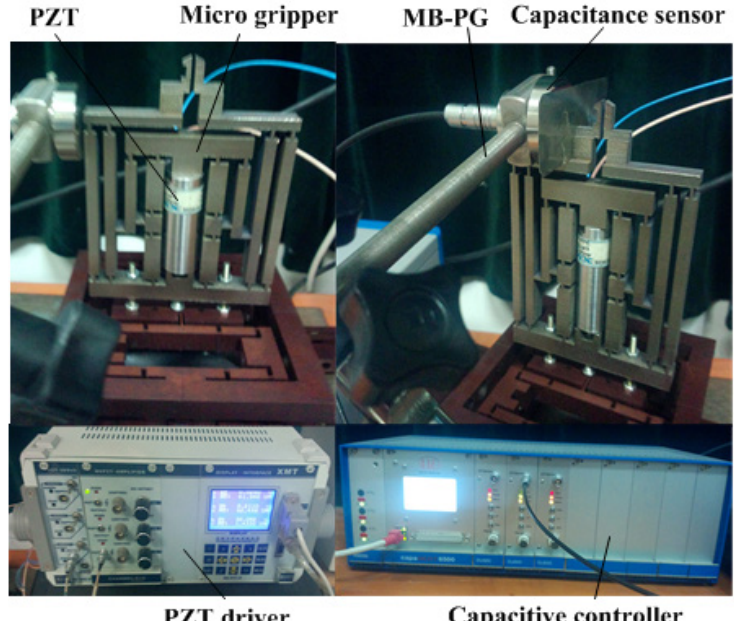

PZT driver

Capacitive controller

Fig. 9. Test of micro gripper

When the input displacement is over $13 \mu \mathrm{m}$, the increment of output displacement is decreasing along with the increasing of input displacement. When the input displacement is over $16 \mu \mathrm{m}$, the PZT is overloaded, the input displacement cannot be increased, so the finite element values, bond graph theoretic values and experimental values of gripper arm output displacement under input displacement of $1 \mu \mathrm{m}$ to $16 \mu \mathrm{m}$ are shown in Fig. 10a, and the maximums are $134.77 \mu \mathrm{m}, 112.6 \mu \mathrm{m}$, and $91.52 \mu \mathrm{m}$, respectively.

Because of the high accuracy, the finite element simulation result is usually considered as the basis of the product performance analysis. The errors between the finite element values and the other two values are shown in Fig. 10b. It can be learned that the error between bond graph theoretic values and 
finite element values is $-(14.01 \%$ to $16.42 \%)$, the error between experimental values and finite element values is $-(21.26 \%$ to $32.09 \%)$, where "-" means the bond graph theoretic values and experimental values are below the finite element values.

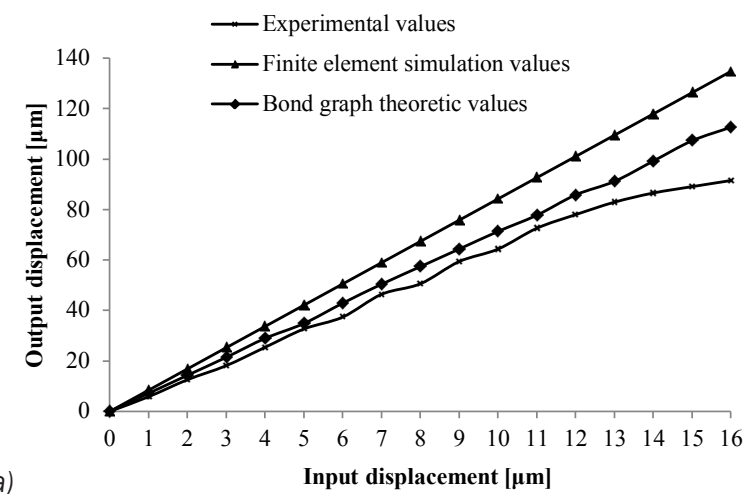

a)
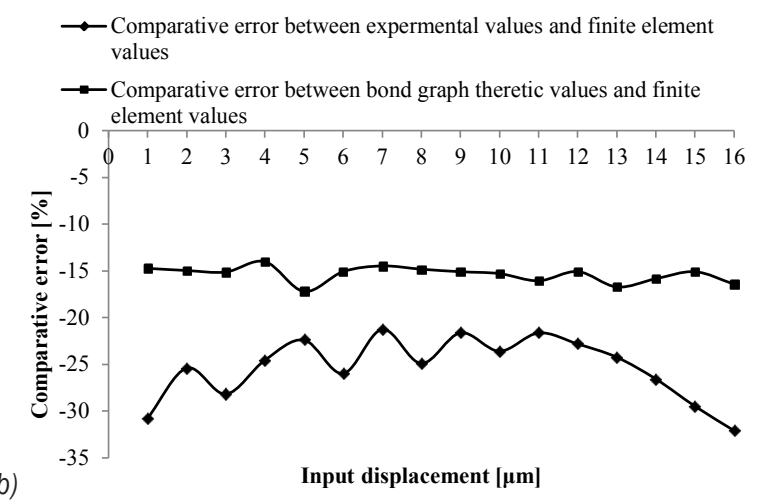

Fig. 10. Output results of micro gripper; a) output displacement, and b) comparative error

Table 4 can be obtained according to the analysis above.

Table 4. Analysis result of micro gripper

\begin{tabular}{lccc}
\hline Micro gripper & Bond graph & Finite element & Experiment \\
\hline One-way magnification & 7.2 & 8.25 & 5.8 \\
\hline Max displacement $[\mu \mathrm{m}]$ & 112.6 & 134.7 & 91.5 \\
\hline Average error [\%] & -13.5 & 0 & -23.4 \\
\hline
\end{tabular}

The experimental values are smaller than the finite element values, and the increase becomes slow when input displacement is $13 \mu \mathrm{m}$, which is different from the theoretical values $18 \mu \mathrm{m}$, that is because the material characteristics have been changed largely by the high-temperature energy of the laser during the procedure of micro gripper. Without heat treatment, the stiffness of micro gripper is influenced by internal stress. The bond graph theoretical values and experimental values are all smaller than the finite element values, and the experimental values are closer to the bond graph theoretical values, so the correctness of the bond graph model of micro gripper and the feasibility of using the band graph method to analyse the fully flexured micro gripper are verified.

\section{CONCLUSIONS}

(1) A three stage amplifying micro gripper has been designed, of which a PRB model has also been set up. The micro gripper is processed based on laser rapid prototyping technology, the stiffness of BATM has been analysed, and the input stiffness of the micro gripper has been derived.

(2) The bond graph model of the micro gripper has been set up, the characteristic equations and state equations of the micro gripper are derived. The input/output displacement simulation curves of the micro gripper and angular displacement simulation curves of the flexible hinge are obtained via Matlab simulation, according to the state equations.

(3) The FEA for the micro gripper is conducted, and the experiments to verify it have been done. Matlab simulation values, FEA values, and experimental values are compared, the Matlab simulation values and FEA values are essentially the same. The error between Matlab simulation values and FEA values is small, which verifies the correctness and feasibility of the established bond graph model of the micro gripper; therefore, the bond graph method provides a new method of analysing the state changes of a fully flexible mechanism and its individual internal components.

In order to make the micro gripper more practical, there still are some tasks for future study:

(1) The research of clamping force and the relevant text need to be done;

(2) The bond graph method used in this paper still need further study for other compliant mechanisms.

\section{ACKNOWLEDGEMENTS}

This work is supported by the National Natural Science Foundation of China (51275537), the Independent Research Fund of State Key Laboratory of Mechanical Transmission (NO. SKLMT-ZZKT-2012 MS 05), the Open Foundation of Shang Hai Key Laboratory of Spacecraft Mechanism (SM2014D201). 


\section{REFERENCES}

[1] Russell, R.A. (1993). Development of a robotic manipulator for micro-assembly operations. Proceedings of the IEEE/ RSJ International Conference on Intelligent Robots and Systems, Yokohama, vol. 1, p. 471-474, D0l:10.1109/ IROS.1993.583143.

[2] Codourey, A., Rodriguez, M., Pappas, I. (1997). A taskoriented teleoperation system for assembly in the microworld. Proceedings of the $8^{\text {th }}$ International Conference on Advanced Robotics, Monterey, p. 235-240, D0I:10.1109/ icar.1997.620188.

[3] Chan, H.Y., Li, W.J. (2003). A thermally actuated polymer micro robotic gripper for manipulation of biological cells. Proceedings of the IEEE International Conference on Robotics and Automation, vol. 1, p. 288-293, Dol:10.1109/ ROBOT.2003.1241610.

[4] Beyeler, F., Bell, D.J., Nelson, B.J., Sun, Y., Neild, A., Oberti, S., Dual, J. (2006). Design of a micro-gripper and an ultrasonic manipulator for handling micron sized objects. International Conference on Intelligent Robots and Systems, p. 772-777, DOI:10.1109/IROS.2006.282628.

[5] Zubir, M.N.M., Shirinzadeh, B. (2009). Development of a high precision flexure-based micro gripper. Precision Engineering, vol. 33, no. 4, p. 362-370, D0l:10.1016/j. precisioneng.2008.10.003.

[6] Kyung, J.H., Ko, B.G., Ha, Y.H., Chung, G.J. (2008). Design of a micro gripper for micromanipulation of microcomponents using SMA wires and flexible hinges. Sensors and Actuators A: Physical, vol. 141, no. 1, p. 144-150, Dol:10.1016/j. sna.2007.07.013.

[7] Ren, H., Gerhard, E. (1997). Design and fabrication of a current-pulse-excited bistable magnetic microactuator. Sensors and Actuators A: Physical, vol. 58, no. 3, p. 259-264, DOI:10.1016/S0924-4247(97)01395-2.

[8] Watson, B., Friend, J., Yeo, L. (2009). Piezoelectric ultrasonic micro/milli-scale actuators. Sensors and Actuators A: Physical, vol. 152, no. 2, p. 219-233, D0l:10.1016/j.sna.2009.04.001.

[9] Nah, S.K., Zhong, Z.W. (2007). A micro gripper using piezoelectric actuation for micro-object manipulation. Sensors and Actuators A: Physical, vol. 133, no. 1, p. 218-224, DOl:10.1016/j.sna.2006.03.014.

[10] Zubir, M.N.M., Shirinzadeh, B., Tian, Y. (2009). Development of a novel flexure-based micro gripper for high precision microobject manipulation. Sensors and Actuators A: Physical, vol. 150, no. 2, p. 257-266, D0l:10.1016/j.sna.2009.01.016.

[11] Ramadan, A.A., Takubo, T., Mae, Y., Oohara, K., Arai, T. (2009). Developmental process of a chopstick-like hybrid-structure two-fingered micromanipulator hand for 3-d manipulation of microscopic objects. IEEE Transactions on Industrial Electronics, vol. 56, no. 4, p. 1121-1134, D0I:10.1109/ TIE.2008.2008753.

[12] Xiao, S.L., Li, Y.M., Zhao, X.H. (2011). Design and analysis of a novel micro-gripper with completely parallel movement of gripping arms. 6th IEEE Conference on Industrial Electronics and Applications, p. 2127-2132, Dol:10.1109/ ICIEA.2011.5975943.
[13] Yu, B.Q., Lei, Z., Weng, H.S. (2011). Optimal design of micro flexible gripper. International Conference on Electric Information and Control Engineering, p. 5154-5157, DOI:10.1109/ICEICE.2011.5776813.

[14] Li, Y., Huang, J., Huang, G., Wang, W., Chen, J., Zeng, Z. (2014). Comparison of radial forging between the two-and threesplit dies of a thin-walled copper tube during tube sinking. Materials \& Design, vol. 56, p. 822-832, D0l:10.1016/j. matdes.2013.11.079.

[15] Jain, R.K., Majumder, S., Ghosh, B., Saha, S. (2015). Design and manufacturing of mobile micro manipulation system with a compliant piezoelectric actuator based micro gripper. Journal of Manufacturing Systems, vol. 35, p. 76-91, D0l:10.1016/j. jmsy.2014.12.001.

[16] Ford, S., Macias, G., Lumia, R. (2015) Single active finger IPMC micro gripper. Smart Materials and Structures, vol. 24, no. 2, DOl:10.1088/0964-1726/24/2/025015.

[17] Hao, Y., Yuan, W., Zhang, H., Kang, H., Chang, H. (2016). A rotary micro gripper with locking function via a ratchet mechanism. Journal of Micromechanics and Microengineering, vol. 26, no. 1, D0l:10.1088/0960-1317/26/1/015008.

[18] Jain, R.K., Majumder, S., Ghosh, B. (2015). Design and analysis of piezoelectric actuator for micro gripper. International Journal of Mechanics and Materials in Design, vol. 11, no. 3 , p. 253-276, DOI:10.1007/s10999-014-9264-z.

[19] Wang, F., Liang, C., Tian, Y., Zhao, X. (2015). Design of a piezoelectric-actuated micro gripper with a three-stage flexure-based amplification. IEEE/ASME Transactions on Mechatronics, vol. 20, no. 5, p. 2205-2213, D0l:10.1109/ TMECH.2014.2368789.

[20] Paynter, H. (1992). An epistemic prehistory of Bond Graphs. in Breedveld, P.C., G. Dauphin-Tanguy, G. (eds.). Bond Graphs for Engineers. Elsevier, Amsterdam, p. 3-17.

[21] Gawthrop, P.J., Bevan, G.P. (2007). Bond-graph modeling. IEEE Control Systems, vol. 27, no. 2, p. 22-45, D0I:10.1109/ MCS.2007.338279.

[22] Yildiz, I., Omurlu, V.E. (2011). Reduced order dynamics and stability of a parallel manipulator through bond-graph technique. XXIII International Symposium on Information, Communication and Automation Technologies, Dol:10.1109/ ICAT.2011.6102084.

[23] Lobontiu, N., Garcia, E. (2003). Analytical model of displacement amplification and stiffness optimization for a class of flexure-based compliant mechanisms. Computers \& Structures, vol. 81, no. 32, p. 2797-2809, D0l:10.1016/j. compstruc.2003.07.003.

[24] Kivi, A.R., Azizi, S. (2015). On the dynamics of a micro-gripper subjected to electrostatic and piezoelectric excitations. International Journal of Non-Linear Mechanics, vol. 77, p. 183-192, D0I:10.1016/j.jinonlinmec.2015.07.012.

[25] Alogla, A.F., Amalou, F., Balmer, C., Scanlan, P., Shu, W., Reuben, R.L. (2015). Micro-tweezers: Design, fabrication, simulation and testing of a pneumatically actuated microgripper for micromanipulation and microtactile sensing. Sensors and Actuators A: Physical, vol. 236, p. 394-404, DOI:10.1016/j.sna.2015.06.032. 